\title{
Evaluation of macular pigment optical density following femtosecond laser-assisted cataract
} surgery

\author{
Tommaso Verdina' \\ Purva Date ${ }^{2}$ \\ Caterina Benatti ${ }^{1}$ \\ Andrea Lazzerini' \\ Elisa Fornasari' \\ Michele De Maria ${ }^{3}$ \\ Elena Pellacani' \\ Matteo Forlini ${ }^{4}$ \\ Gian Maria Cavallini' \\ 'Institute of Ophthalmology, University \\ of Modena and Reggio Emilia, Modena, \\ Italy; ${ }^{2}$ Aditya Jyot Eye Hospital, Mumbai, \\ India; ${ }^{3}$ Clinical and Experimental \\ Medicine PhD Program, University of \\ Modena and Reggio Emilia, Modena, Italy; \\ ${ }^{4}$ Domus Nova Hospital, Ravenna, Italy
}

This article was published in the following Dove Press journal: Clinical Ophthalmology

Background: To evaluate macular pigment optical density (MPOD) after bimanual femtosecond laser-assisted cataract surgery (FLACS) compared to standard bimanual phacoemulsification (B-MICS).

Methods: A prospective, case matched, comparative cohort study conducted at the Institute of Ophthalmology, University of Modena and Reggio Emilia (Italy); 30 eyes underwent bimanual FLACS with low-energy Ziemer LDV Z8 (FLACS) and 30 underwent B-MICS standard technique (B-MICS). All interventions were conducted by the same expert surgeon. MPOD using the Macular Pigment Screener II (MPS II) was evaluated at baseline, 7 and 30 days after surgery. As secondary outcomes, we considered best corrected visual acuity (BCVA) and central macular thickness (CMT) obtained using optical coherence tomography.

Results: In all cases, a BunnyLens AF IOL was safely implanted in the capsular bag through a $1.4 \mathrm{~mm}$ incision. We found a significant reduction in MPOD in both groups at 7 and 30 days; 0.16 \pm 0.14 and $0.10 \pm 0.12$ (FLACS) and $0.18 \pm 0.13$ and $0.15 \pm 0.14$ (B-MICS), respectively $(P<0.05$ ). However, there was no significant difference between the two groups at either $7(P=0.52)$ or 30 days $(P=0.18)$. BCVA improved significantly in both groups and CMT increased in both groups $(P<0.001, P<0.001$, respectively). BCVA and CMT were similar between the groups with a significant difference in CMT in favor of the FLACS group at 30 days $(P=0.017)$.

Conclusions: MPOD was reduced in both groups without any significant difference between the FLACS and B-MICS cataract interventions. FLACS is associated with a significantly higher increase of macular thickness at 30 days compared to B-MICS.

Keywords: femtosecond laser, macular pigment, macular pigment optical density (MPOD), femtosecond laser-assisted cataract surgery (FLACS), bimanual microincision cataract surgery (B-MICS), flicker photometry

\section{Background}

Macular pigment (MP) is made up of retinal carotenoids like lutein and zeaxanthin and protects the retina against photo-oxidative damage. These components absorb blue light in the inner retina and act as free radical scavengers for the photoreceptor cells. ${ }^{1}$ MP is deposited mainly in the Henle fiber layer in the fovea and in the inner plexiform layers at the parafoveal site of the retina with concentration peaks in the center of the macula, decreasing progressively moving to the periphery. ${ }^{2}$

Detecting the level of macular pigment optical density (MPOD) is an innovative approach to measure the density of MP in the macular area. ${ }^{3}$ Different methods have been investigated to calculate MPOD such as motion-detection photometry,
Institute of Ophthalmology, University of Modena and Reggio Emilia, Via del Pozzo 7I, 41100 Modena, Italy

Tel +3959360309

Fax +3959371532

Email tommaso.verdina@gmail.com 
one-wavelength reflection fundus imaging, raman spectrometry, auto-fluorescence spectrometry and heterochromatic flicker photometry (HFP). The macular pigment screener II (MPSII® Elektron Technology, Switzerland) is a recent innovation for the measurement of MPOD. ${ }^{4}$ This is a macular densitometer that works with HFP technique and detects the blue light absorption rate of MP; this examination is simple to perform and the results are easy to interpret. Recent studies investigated the usefulness of this device, and it has been shown that a low MPOD is a key risk factor for age-related macular disease (AMD). ${ }^{5}$ In contrast, a high MPOD helps to improve visual performance by reducing glare and giving better contrast sensitivity and visual acuity. ${ }^{6}$

Cataract surgery is a commonly performed ophthalmic surgical procedure and can have influence on the MPOD. In particular, it has been previously reported that excessive light exposure and microscope photo-oxidative stress are inversely proportional with MPOD and, following cataract surgery, there is an increased short-wavelength light transmission to the retina with an increased risk of AMD development. $^{7,8}$

Femtosecond laser-assisted cataract surgery (FLACS) is a new approach in assisting the surgeon in performing a controlled and reproducible capsulotomy, an efficient nucleus fragmentation and precise clear corneal incisions for cataract surgery. ${ }^{9}$ The combination of FLACS with a bimanual technique has been proven to be safe and effective. ${ }^{10-13}$ However, it is known that this technique produces higher postoperative inflammation, in some cases with resultant effect on final visual recovery and patient satisfaction. ${ }^{14,15}$ Moreover, FLACS procedure takes longer surgical time with exposition of the macula to a higher surgical stress: the laser procedures (including suction, tissue photodisruption, bubble scattering) could be transmitted to the retina creating a retinal injury ${ }^{16}$ though influencing MP density.

Understanding the relationship between MPOD and cataract surgery could be of particular interest in the prevention of AMD after cataract surgery. Recent studies showed that mean MPOD values were found to be statistically significantly lower in patients who had undergone cataract surgery. ${ }^{17,18}$ To the best of our knowledge, there are no studies available in the literature investigating the effect of FLACS on MPOD.

The aim of our study was to compare the changes in MPOD assessed with MPSII, following FLACS compared to standard B-MICS technique for cataract surgery. The study secondary outcomes included best corrected visual acuity (BCVA) and central macular thickness (CMT) measured with optical coherence tomography (OCT).

\section{Material and methods}

This prospective, case matched, comparative cohort study was conducted at the Institute of Ophthalmology, University of Modena and Reggio Emilia from January 2017 to June 2017. The study was approved by the local Ethics Committee of the University of Modena and Reggio Emilia and was conducted according to the Declaration of Helsinki. All patients gave written informed consent.

The study design comprised two groups of consecutive patients treated for cataract surgery with either the FLACS technique (FLACS) on the day in which the femtosecond laser was available, or the standard B-MICS technique (B-MICS), on all the other days, by the same experienced surgeon (GMC). Patients were selected from according to cataract nuclear sclerosis grade 1 (NS1) and 2 (NS2) according to the Lens Opacities Classification System III (LOCS III). ${ }^{19}$ Exclusion criteria included diabetes, any previous ocular surgery, posterior segment pathologies (eg, AMD, diabetic retinopathy), LOCS $\geq \mathrm{NS} 3$, preexisting ocular pathologies like uveitis, glaucoma, corneal opacities, poor mydriasis $(<4 \mathrm{~mm})$, low endothelial cell count $\left(<1500\right.$ cells $\left./ \mathrm{mm}^{2}\right)$, monocular patients, advanced age $(>75$ years old) and patients unable to collaborate during the MPOD evaluation.

All interventions were performed using the same phaco-emulsifying machine (Faros, Oertli Instruments AG, Berneck, Switzerland).

A detailed history and ophthalmic examination were performed in all patients preoperatively including anterior segment biomicroscopy and fundus examination with a slit-lamp assessment, CMT with spectral domain OCT (OCT SLO, Optos, Scotland, UK), BCVA with Snellen charts and analyzed with the LogMar methodology, axial length and biometry with IOL Master (Carl Zeiss Meditech, Jena, Germany) and MPOD with the MPS II (Elektron Technology) (Figure 1).

MPOD measurements were performed three times prior to pupil dilatation by the same trained investigator, and mean values of collected data were used. During the exam, two different wavelength flicker lights (465 nm blue light, absorbed by the MP and $530 \mathrm{~nm}$ green light, not absorbed by the MP) were alternatively presented to the 


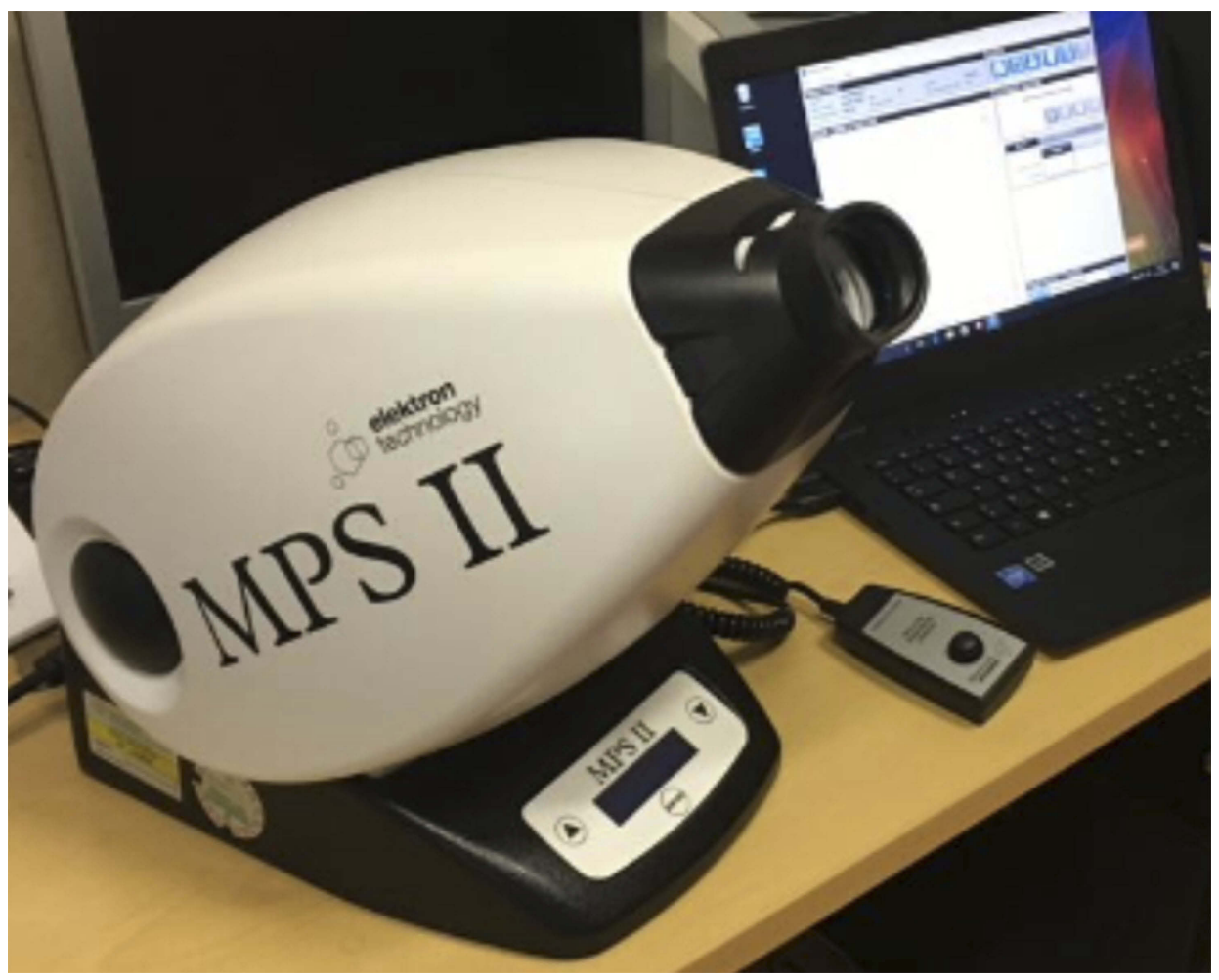

Figure I Macular pigment screener II (MPSII® Elektron Technology, Switzerland).

patient, who was instructed to push a button as soon as a flicker perception was detected. MPS II studied the parafoveal and foveal threshold and automatically calculated MPOD studying the difference in the intensity of illumination between blue-green ratios. ${ }^{20}$

MPOD results are provided on a scale of $0-1$, where " 0 " (maximum level of blue light hitting the macula) corresponds to a higher risk of developing age-related macular degeneration (AMD), while "1.0" indicates a protective factor and low risk of $\mathrm{AMD}$ development.

For CMT evaluation the OCT acquisition protocol consisted of a macular cube $512 \times 128$ scan pattern in which a $6.0 \times 6.0 \mathrm{~mm}$ region of the retina was scanned (a total of 65.536 sampled points) and 5 raster horizontal lines which passed through fovea. Macular thickness value was obtained using the integrated OCT software.

Patients were prescribed a NSAID eye drops preoperatively 3 times a day for 3 days before surgery in both groups. Peri-operative asepsis included topical ofloxacin eyedrop, 5\% povidone-iodine in the conjunctival cul-desac, $10 \%$ povidone-iodine on the periocular skin, careful sterile draping of the eyelid margins and eyelashes. During surgical maneuvers, Vancomycin was added to the irrigating solution. At the end of the surgery, intracameral and topical antibiotic were applied.

Standard B-MICS and bimanual FLACS surgery techniques have been previously described. ${ }^{11,12}$ Surgeon planned to implant the same model of hydrophilic acrylic BunnyLensAF (Hanita Lenses, Israel) IOL in all eyes.

Total surgery time was recorded as well as effective phacoemulsification time (EPT). Total surgery time for FLACS was calculated from the application of the disposable suction ring in the cornea to corneal microincisions' hydrosuture subtracting the time between end of the suction ring removal to the opening of the first corneal incision; B-MICS total surgery time was calculated from the first corneal incision to corneal hydrosuture.

Post-operative values were recorded at 7 and 30 days after surgery, in terms of MPOD, BCVA, and CMT.

Data were collected with an Excel database (Microsoft Excel 2010, Microsoft Office Professional Plus 2010; Microsoft Corporation, Redmond, WA, USA) and Stata 13.1 software (StataCorp LP, College Station, TX, USA) was employed for Student's $t$-test and Wilcoxon rank sum test. A $P$-value $<0.05$ was considered statistically significant. All results were expressed as mean \pm standard deviation. 


\section{Results}

FLACS included 30 eyes from 17 patients with a mean age of $72.10 \pm 12.03$ years, and the case matched B-MICS group included 30 eyes from 20 patients with a mean age of $70.11 \pm 9.3$ years. FLACS included mean cataract LOCS value of $1.57 \pm 0.50$ compared to B-MICS values of $1.63 \pm 0.49, P=0.66$. (Table 1 )

A significantly longer total surgical time was observed for FLACS; $15.88 \pm 2.63$ mins vs $12.69 \pm 2.79$ mins, $P<0.05$. The mean EPT was significantly shorter for FLACS $(3.62 \pm 1.74 \mathrm{~s})$ compared to B-MICS (4.22 $\pm 1.84 \mathrm{~s}), P=0.04$.

The surgeon implanted the IOL in all eyes successfully in the capsular bag. No major intraoperative complications were recorded. No cystoid macular edema was reported in both groups.

Table I Demographic data at baseline, total surgical time and effective phaco time (EPT) for both groups

\begin{tabular}{|l|l|l|l|}
\hline & $\begin{array}{l}\text { Group A } \\
\text { (FLACS) }\end{array}$ & $\begin{array}{l}\text { Group B } \\
\text { (B-MICS) }\end{array}$ & P-value \\
\hline Eyes (n) & 30 & 30 & - \\
Mean age (y) \pm SD & $72.10 \pm 12.03$ & $70.11 \pm 9.3$ & $>0.05$ \\
Right/left eyes (n) & $13 / 17$ & $16 / 14$ & $>0.05$ \\
Male/female (n) & $7 / 10(17)$ & $9 / 11(20)$ & $>0.05$ \\
Cataract grade & $1.57 \pm 0.50$ & $1.63 \pm 0.49$ & 0.66 \\
(LOCS III) & $15.88 \pm 2.63$ & $12.69 \pm 2.79$ & $>0.05$ \\
Total surgical & & & \\
time (mins) & $3.62 \pm 1.74$ & $4.22 \pm 1.84$ & 0.04 \\
EPT (s) & & \\
\hline
\end{tabular}

Abbreviations: FLACS, femtosecond laser-assisted cataract surgery; LOCS, Lens Opacities Classification System III.
There was a statistically significant reduction of MPOD at both 7 and 30 days post-operatively $(P<0.05)$ for both groups (Figure 2). FLACS MPOD reduced by $0.16 \pm 0.14$ at 7 days and $0.10 \pm 0.12$ at 30 days, and B-MICS MPOD reduced by $0.18 \pm 0.13$ and $0.15 \pm 0.14$, respectively. The difference in MPOD reduction among the groups was not significant.

The mean preoperative BCVA for the FLACS group was $0.480 \pm 0.193 \mathrm{LogMar}$, which significantly improved to $0.020 \pm 0.032 \mathrm{LogMar}$ at 30 days follow-up $(P<0.05)$. The mean preoperative BCVA for the B-MICS group was $0.503 \pm 0.166$ LogMar also significantly improved to $0.027 \pm 0.035$ LogMar at 30 days follow-up $(P<0.05)$. The difference among the groups was not statistically significant (Figure 3).

CMT increase was statistically significant in both groups $(P<0.05)$ with a statistically significant difference in change in CMT in favor of FLACS when compared with B-MICS at 30 days $(P=0.017)$ (Figure 4$)$.

All post-operative results are reported in Tables 2 and 3.

\section{Discussion}

The introduction of FLACS has led to considerable advantages in cataract surgery including increased precision in anterior capsulotomy, formation of corneal incisions and lens fragmentation, leading to a reduction in the use of ultrasound energy. According to recent literature, it is demonstrated that FLACS and standard phacoemulsification do not differ in terms of complications, safety, refractive outcomes and general patient satisfaction. ${ }^{11}$ A recent study found that mean MPOD values were statistically significantly lower

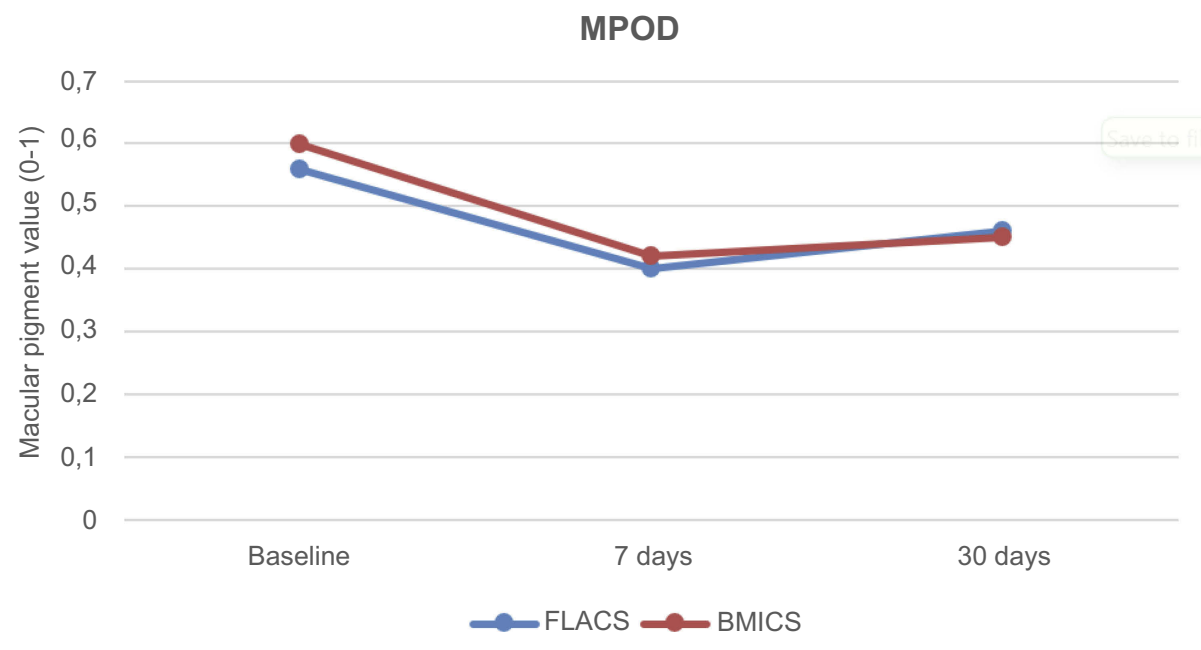

Figure 2 Macular pigment optical density variation during the follow-up.

Abbreviations: B-MICS, bimanual phacoemulsification; FLACS, femtosecond laser-assisted cataract surgery; MPOD, macular pigment optical density. 


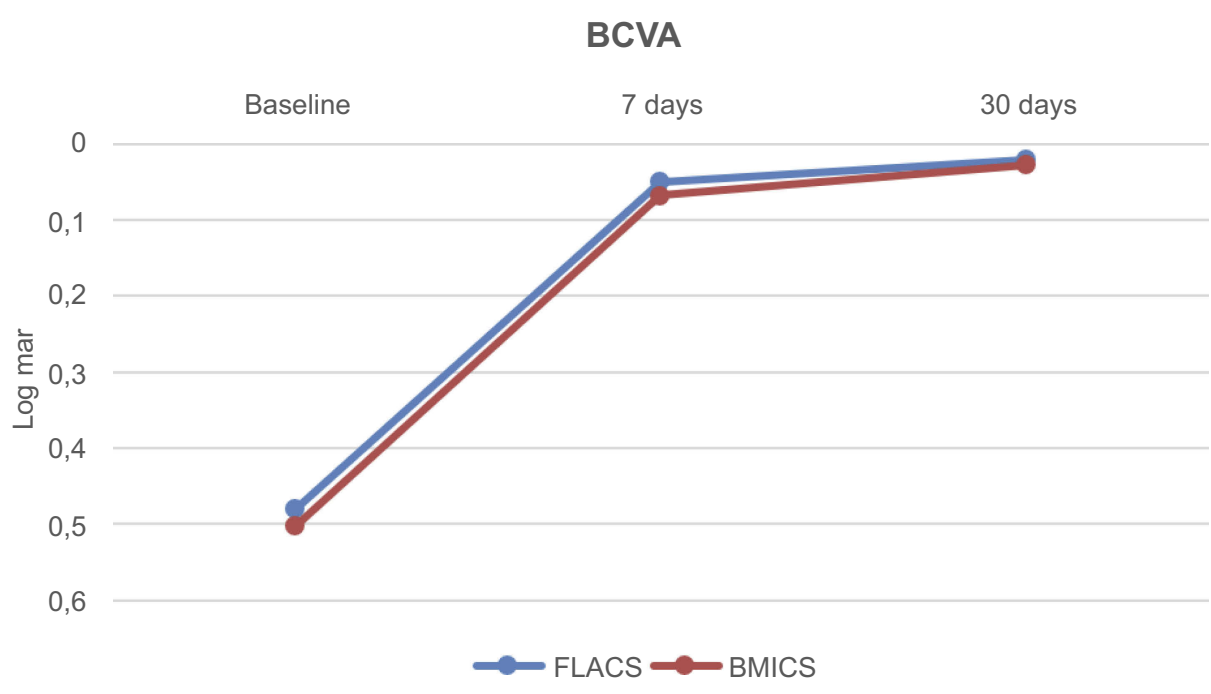

Figure 3 Best corrected visual acuity variation during the follow-up.

Abbreviations: BCVA, best corrected visual acuity; B-MICS, bimanual phacoemulsification; FLACS, femtosecond laser-assisted cataract surgery.

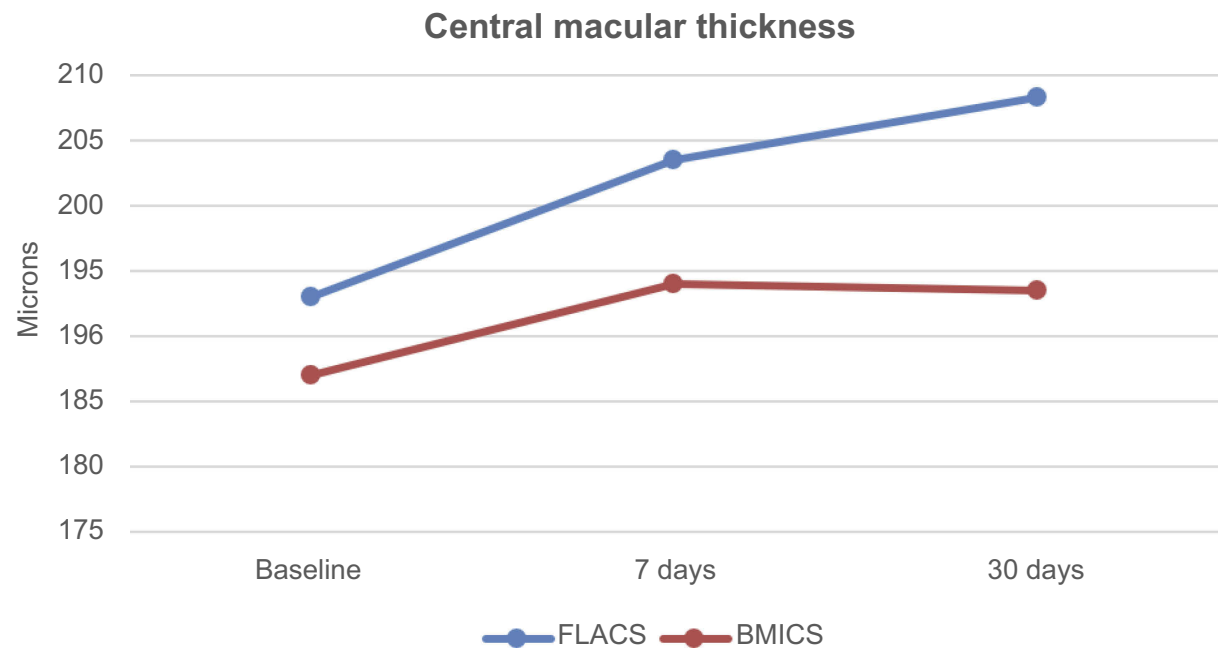

Figure 4 Central macular thickness (CMT) variation during the follow-up.

Abbreviations: B-MICS, bimanual phacoemulsification; FLACS, femtosecond laser-assisted cataract surgery.

following cataract surgery compared to an age-matched group subjects without previous cataract surgery. ${ }^{17}$

When the cataract is removed a dramatic increase in the ocular transmittance of radiation occurs with an augmented exposure of the retina to blue light and to a portion of the UVA radiation thus to oxidative-stress. As long as the short-wavelength radiation strikes the retina a cascade of cellular effects is induced and some clinical reports suggested that cataract surgery could increase the development or progression of AMD. ${ }^{7,8}$ However, no studies have investigated yet on the effect of FLACS procedure on the MPOD variation.
In our study, MPOD was found to be statistically significantly decreased 7 days after both the FLACS and B-MICS interventions $(P<0.05)$, but there was no significant difference between the two groups (Tables 2 and 3 ). This data suggests that prolonged exposure to the light of the surgical microscope may negatively influences the MPOD, without any correlation to a particular technique.

In both groups, a photo-oxidative stress, comparable to that generated by the action of blue light, could have been generated at the retinal photoreceptors and may be responsible for the reduction of MPOD in the immediate postoperative period. 
Table 2 Mean values of preoperative and postoperative parameters for Group A and Group B

\begin{tabular}{|l|l|l|l|}
\hline & $\begin{array}{l}\text { Group A } \\
\text { (FLACS) }\end{array}$ & $\begin{array}{l}\text { Group B } \\
\text { (B-MICS) }\end{array}$ & P-value \\
\hline MPOD & & $0.60 \pm 0.15$ & $P=0.30$ \\
Preop & $0.56 \pm 0.18$ & $0.42 \pm 0.13$ & \\
7 days & $0.40 \pm 0.19$ & $0.45 \pm 0.16$ & \\
30 days & $0.46 \pm 0.18$ & & \\
\hline BCVA & & $0.503 \pm 0.166$ & $P=0.63$ \\
Preop & $0.480 \pm 0.193$ & $0.068 \pm 0.047$ & \\
7 days & $0.050 \pm 0.052$ & $0.027 \pm 0.035$ & \\
30 days & $0.020 \pm 0.032$ & & \\
\hline CMT & & $187.00 \pm 22.50$ & $P=0.34$ \\
Preop & $193.00 \pm 18.41$ & $194.00 \pm 27.74$ & \\
7 days & $203.50 \pm 24.25$ & $193.50 \pm 28.56$ & \\
30 days & $208.33 \pm 24.93$ & & \\
\hline
\end{tabular}

Abbreviations: MPOD: macular pigment optical density. BCVA expressed in logMAR. CMT: central macular thickness, expressed in microns; BCVA, best corrected visual acuity; FLACS, femtosecond laser-assisted cataract surgery; B-MICS, bimanual phacoemulsification.

Our results confirm the results of previous investigations reporting a reduction of MPOD after cataract surgery. ${ }^{17,18}$

In recent study Gultekin et $\mathrm{al}^{21}$ stated that MPOD levels significantly decrease in pseudophakic eyes operated with traditional phacoemulsification technique while duration of postoperative period indicated an increase; however, in this study, there is no comparison between preoperative and postoperative values.

In our study, we found that MPOD slightly increased at the 30-day follow-up in both groups without any significant differences between the two groups; however, MPOD at 30-day follow-up did not reached pre-operative values $(P>0.05)$. In the FLACS group, we noticed a lower MPOD decrease than in the B-MICS group, but a significant difference was not found.

Preoperative BCVA was comparable in both groups $(P>0.05)$ and improved significantly in both groups during the follow up period, supporting previous literature suggesting that both surgical procedures are both effective and safe in terms of post-operative visual rehabilitation.

Additionally, CMT was comparable in both groups preoperatively $(P=0.34)$ and increased significantly after 30 days following the intervention in both groups. The increase was statistically higher in the FLACS group compared to the B-MICS group.

Recent literature has described heterogeneous results on this topic: some authors reported no significant differences in the post-operative follow-up in patients undergoing FLACS and conventional MICS cataract surgery $^{22}$ while other studies have reported a higher post-operative CMT in patients who underwent cataract extraction with FLACS compared to conventional MICS. ${ }^{23}$ Some authors have affirmed the importance of a long learning surgical curve to overcome intra- and post-operative complications, especially for postoperative macular edema. ${ }^{24}$ In the current study the same surgeon had extensive experience for both the FLACS and the B-MICS techniques.

In our case series, we report higher CMT in the FLACS group. This result may be a result of a higher degree of inflammation produced by femtosecond laser, which provokes the release of intraoperative prostaglandins. ${ }^{9,10}$ However, no changes in the BCVA reported in this study among the two groups affirms that both techniques have excellent intra- and postoperative reliability.

Table 3 Comparison of the differences for each considered parameter for both groups

\begin{tabular}{|l|l|l|l|}
\hline & Group A (FLACS) & Group B (BMICS) & $P$-value (A vs B) \\
\hline MPOD & & & \\
7 days & $0.16 \pm 0.14(-28.6 \%)$ & $0.18 \pm 0.13(-30 \%)$ & $0.15 \pm 0.14(-25 \%)$ \\
30 days & $0.10 \pm 0.12(-17.9 \%)$ & & $P=0.52$ \\
\hline BCVA & & $0.435 \pm 0.166$ & $P=0.18$ \\
7 days & $0.429 \pm 0.192$ & $0.476 \pm 0.168$ & $P=0.99$ \\
30 days & $0.459 \pm 0.196$ & & $P=0.84$ \\
\hline CMT & & $7.00 \pm 10.95(+3.7 \%)$ & \\
7 days & $10.50 \pm 16.42(+5.4 \%)$ & $6.50 \pm 11.46(+3.4 \%)$ & $P=0.36$ \\
30 days & $15.33 \pm 15.02(+7.9 \%)$ & $P=0.017$ \\
\hline
\end{tabular}

Abbreviations: B-MICS, bimanual phacoemulsification; BCVA, best corrected visual acuity; CMT, central macular thickness; FLACS, femtosecond laser-assisted cataract surgery; MPOD, macular pigment optical density. 
This cohort study is limited by the small number of participants and the relatively short follow-up that could have affected the results. Moreover, patients were not randomized.

In conclusion, our study proves that MPOD decreases following cataract surgery with no differences observed between the FLACS or the B-MICS techniques. BCVA and CMT outcomes were also similar, with an increase in CMT observed in the FLACS group. Further studies with a larger study population and longer follow-up may be needed to confirm these findings.

\section{Data sharing statement}

The datasets used and/or analyzed during the current study are available from the corresponding author on reasonable request.

\section{Acknowledgments}

The authors would like to thank Johanna Chester for her critical revision of content and editorial assistance.

\section{Author contributions}

All authors contributed to data analysis, drafting and revising the article, gave final approval of the version to be published, and agree to be accountable for all aspects of the work.

\section{Disclosure}

The authors report no conflicts of interest in this work.

\section{References}

1. Seddon JM, Ajani UA, Sperduto RD, et al; Dietary carotenoids, vitamins $\mathrm{A}, \mathrm{C}$, and $\mathrm{E}$, and advanced age-related macular degeneration. Eye Disease Case-Control Study Group. JAMA. 1994;272(18):1413-1420.

2. Trieschmann M, van Kuijk FJ, Alexander R, et al. Macular pigment in the human retina: histological evaluation of localization and distribution. Eye (Lond). 2008;22:132-137. doi:10.1038/sj. eye. 6702780

3. Bartlett H, Howells O, Eperjesi F. The role of macular pigment assessment in clinical practice: a review. Clin Exp Optom. 2010;93 (5):300-308. doi:10.1111/j.1444-0938.2010.00499.x

4. Ozawa Y, Shigeno Y, Nagai N, et al. Absolute and estimated values of macular pigment optical density in young and aged Asian participants with or without age-related macular degeneration. BMC Ophthalmol. 2017;17(1):161. doi:10.1186/s12886-017-0557-5

5. Arunkumar R, Calvo CM, Conrady CD, Bernstein PS. What do we know about the macular pigment in AMD: the past, the present, and the future. Eye (Lond). 2018;32(5):992-1004. doi:10.1038/s41433018-0044-0

6. Stringham JM, Stringham NT, O'Brien KJ. Macular carotenoid supplementation improves visual performance, sleep quality, and adverse physical symptoms in those with high screen time exposure. Foods. 2017;6(7). doi:10.3390/foods6080062
7. Stringham JM, O’Brien KJ, Stringham NT. Macular carotenoid supplementation improves disability glare performance and dynamics of photostress recovery. Eye Vis (Lond). 2016;3:30. doi:10.1186/s40662-016-0060-8

8. Ciulla TA, Hammond BR, Yung CW, Pratt LM. Macular pigment optical density before and after cataract extraction. IOVS. 2001;42:1338-1341.

9. Grewal DS, Schultz T, Basti S, Dick HB. Femtosecond laser-assisted cataract surgery - current status and future directions. Surv Ophthalmol. 2016;61(2):103-131. doi:10.1016/j.survophthal.2015.09.002

10. Cavallini GM, Verdina T, De Maria M, et al. Bimanual microincision cataract surgery with implantation of the new Incise ${ }^{\circledR}$ MJ14 intraocular lens through a $1.4 \mathrm{~mm}$ incision. Int J Ophthalmol. 2017;10 (11):1710-1715. doi:10.18240/ijo.2017.11.12

11. Cavallini GM, Verdina T, De Maria M, Fornasari E, Volpini E, Campi L. Femtosecond laser-assisted cataract surgery with bimanual technique: learning curve for an experienced cataract surgeon. Int Ophthalmol. 2017. [Epub ahead of print]. doi:10.1007/s10792017-0776-Z

12. Pisciotta A, De Maria M, Verdina T, Fornasari E, de Pol A, Cavallini GM. Anterior capsule of the lens: comparison of morphological properties and apoptosis induction following FLACS and standard phacoemulsification surgery. Biomed Res Int. 2018;2018:7242837. doi:10.1155/2018/7242837

13. Cavallini GM, Fornasari E, De Maria M, Lazzerini A, Campi L, Verdina T. Bimanual femtosecond laser-assisted cataract surgery compared to standard bimanual phacoemulsification: a case-control study. Eur J Ophthalmol. 2018;1120672118805323. Epub 2018 Oct 14.

14. Toto L, Curcio C, Mastropasqua A, et al. Immunohistochemical study of corneal inflammation after femtosecond laser clear corneal incisions or manual surgery. J Cataract Refract Surg. 2016;42 (11):1649-1659. doi:10.1016/j.jcrs.2016.08.031

15. Popovic M, Campos-Möller X, Schlenker MB, Ahmed II. Efficacy and safety of femtosecond laser-assisted cataract surgery compared with manual cataract surgery: a meta-analysis of 14 567 eyes. Ophthalmology. 2016;123(10):2113-2126. doi:10.1016/ j.ophtha.2016.07.005

16. Wang J, Sramek C, Paulus YM, et al. Retinal safety of near-infrared lasers in cataract surgery. J Biomed Opt. 2012;17(9):95001. doi:10.1117/1.JBO.17.9.095001

17. Demirel S, Bilici S, Batıoglu F, Ozmert E. The effect of age and cataract surgery on macular pigment optic density: a cross-sectional, comparative study. Graefes Arch Clin Exp Ophthalmol. 2014;252 (2):213-218. doi:10.1007/s00417-013-2424-2

18. Komar B, Rauscher FG, Wiedemann R, Dawczynski J. Macular pigment optical density measurements by one-wavelength reflection photometry - influence of cataract surgery on the measurement results. Graefes Arch Clin Exp Ophthalmol. 2014;252 (11):1717-1727. doi:10.1007/s00417-014-2627-1

19. Chylack LT Jr1, Wolfe JK, Singer DM, et al; The Longitudinal Study of Cataract Study Group. The lens opacities classification system III. Arch Ophthalmol. 1993;111(6):831-836.

20. Van der Veen RL, Berendschot TT, Hendrikse F, Carden D, Makridaki M, Murray IJ. A new desktop instrument for measuring macular pigment optical density based on a novel technique for setting flicker thresholds. Ophthalmic Physiol Opt. 2009;29 (2):127-137. doi:10.1111/j.1475-1313.2008.00618.x

21. Gultekin BP, Bulut A, Bilen N, Kalayci D. Comparison of macular pigment optical density between phakic and pseudophakic eyes. Biomed Res. 2018;29(8):1653-1656. doi:10.4066/biomedicalresearch.29-17-3631

22. Levitz L, Reich J, Roberts TV, Lawless M. Incidence of cystoids macular edema: femtosecond laser- assisted cataract surgery versus manual cataract surgery. J Cataract Refract Surg. 2015;41:683-686. doi:10.1016/j.jcrs.2014.11.039 
23. Nagy ZZ, Ecsedy M, Kovács I, et al. Macular morphology assessed by optical coherence tomography image segmentation after femtosecond laser-assisted and standard cataract surgery. $J$ Cataract Refract Surg. 2012;38(6):941-946. doi:10.1016/j. jcrs.2012.02.031
24. Roberts TV, Lawless M, Bali SJ, Hodge C, Sutton G. Surgical outcomes and safety of femtosecond laser cataract surgery: a prospective study of 1500 consecutive cases. Ophthalmology. 2013;120 (2):227-233. doi:10.1016/j.ophtha.2012.10.026

\section{Publish your work in this journal}

Clinical Ophthalmology is an international, peer-reviewed journal covering all subspecialties within ophthalmology. Key topics include: Optometry; Visual science; Pharmacology and drug therapy in eye diseases; Basic Sciences; Primary and Secondary eye care; Patient Safety and Quality of Care Improvements. This journal is indexed on PubMed
Central and CAS, and is the official journal of The Society of Clinical Ophthalmology (SCO). The manuscript management system is completely online and includes a very quick and fair peer-review system, which is all easy to use. Visit http://www.dovepress.com/ testimonials.php to read real quotes from published authors. 